\title{
Endoscopic Evaluation of Normal Duodenum in 60 Healthy Dogs
}

\author{
D.U. Lokhande, L.B. Sarkate and G.U. Yadav* \\ Department of Surgery and Radiology, Bombay Veterinary College, Parel, Mumbai 12 \\ (Maharashtra Animal and Fishery Sciences University, Nagpur-440006), India \\ *Corresponding author
}

\section{Keywords}

Duodenoscopy, esogastrointestinal fiberscope, duodenal papilla

Article Info

Accepted:

12 October 2018

Available Online:

10 November 2018

\section{A B S T R A C T}

Diagnostic imaging allows visual evaluation of normal and abnormal morphology of the region and thus helps in pinpoint diagnosis of many diseases. But to identify the abnormality one has to be familiar with the normal anatomy of the system. Duodenoscopy of 60 clinically healthy dogs were performed by esogastrointestinal fiberscope and the appearance such as color of mucous membrane, presence of erythema, erosion and sub mucosal blood vessels on duodenal wall were evaluated. In procedure, the insertion tube was first passed directly into the duodenum through pylorus before actual examination of stomach. In order to pass the tip of scope in the pylorus, the pylorus was first fix carefully at the centre of endoscopic field and a gentle pressure was applied with rotation of both knobs simultaneously in counter clockwise direction. The color of mucosa of duodenum varied from pinkish red (38 cases) to yellowish red (22 cases) and it had velvety texture. Bile was noted in the duodenum in 16 cases, while sub mucosal blood vessels were visualized in two cases. However, erythema or erosion was not seen in any of the cases in this study.The mean distance of Junction of descending and ascending duodenum from upper incisor was measured as $69.15 \pm 1.39,100.85 \pm 0.56$ and $103.85 \pm 0.51 \mathrm{~cm}$ in small, medium and large sized dogs, respectively. On the basis of observations it could be said that working length of 100 and $110 \mathrm{~cm}$ would be suitable for small and medium sized dogs, respectively. The use of esophagogastroduodenoscope with working length of 110 to $120 \mathrm{~cm}$ would be more appropriate for large sized dogs.

\section{Introduction}

The love and affection to pet animal has increased by many folds as they are considered as members of family and therefore qualified clients are insisting for the use of safe noninvasive modern imaging techniques for the prompt diagnosis for their valuable pets. Diagnostic imaging allows visual evaluation of normal and abnormal morphology of the region and thus helps in pinpoint diagnosis of many diseases. But to identify the abnormality, one has to be familiar with the normal anatomy of the system.

Endoscopy is one of the non-invasive, a traumatic and more sensitive tool for detection of morphological alteration in the organs. The techniques of endoscopic diagnosis and 
treatment of gastrointestinal tract diseases has been recently reestablished in veterinary medicine. Various workers are performing accurate diagnosis successfully by endoscopy but the basic knowledge and normal finding of duodenoscopy is still limited. Therefore the study was performed and endoscopic view of normal duodenum was recorded and evaluated

\section{Materials and Methods}

The study was conducted on total 60 clinical cases presented for various operations such as spaying, castration, small growths and simple wounds. The selection was done on the basis of physical examination and evaluation of blood for CBC, LFT and KFT and the dogs with normal values were selected for duodenoscopy. The duodenoscopy of all above normal and healthy dogs were performed by esogastrointestinal fiberscope. During the endoscopy, dogs were sedated with triflupromazine hydrochloride $(1 \mathrm{mg} / \mathrm{kg}, \mathrm{i.v})$ or acepromazine maleate $(0.05 \mathrm{mg} / \mathrm{kg}, \mathrm{i.v}), 10$ minutes prior to the intravenous administration of thiopentone sodium (20 $\mathrm{mg} / \mathrm{kg}$., i.v) or propofol $(5 \mathrm{mg} / \mathrm{kg}$. i.v) for induction of anaesthesia. The procedure of duodenoscopy was performed as described by Tams (1999) and the endoscopic images of normal healthy structure were recorded and documented. During the duodenoscopy, the texture and color of mucous membrane of duodenum were recorded. Similarly presences of erythema, erosion and sub mucosal blood vessels in the duodenal wall were evaluated and recorded. After insertion of endoscope in duodenum, the distance of different parts of the tract like proximal duodenum, junction of descending and ascending duodenum and major duodenal papillae from upper incisors were also measured and recorded in different sizes of dogs. The length of insertion tube required for duodenoscopy in different sizes of dogs were also calculated based on the distance of pylorus or the junction of descending and ascending duodenum from upper incisors measured in different sizes of dogs.

\section{Results and Discussion}

The procedure of duodenoscopy was found little difficult to perform, specially the insertion of tube in pylorus. During the procedure, the dog was kept in left lateral recumbent position in order to settle the materials in the fundus and body of stomach. The mouth was kept open during procedure by a stainless steel tube fixed on upper and lower canine teeth of one side of the jaw. This provided more working space for smooth and easy passage of insertion tube through oral cavity.

The insertion of the tube in pylorus and duodenum required a good practice as well as understanding the physiology of stomach wall. Generally during the examination of stomach, continuous insufflations and manipulation is required which ultimately resulted in physiological closure of pylorus in response to gastric distension and more contractile action of the antrum during gastroscopy (Tams, 1999). Therefore, the insertion tube was first passed directly into the duodenum through pylorus before actual examination of stomach.

In order to pass the tip of scope in the pylorus, the pylorus was first fix carefully at the centre of endoscopic field (Fig. 1) and a gentle pressure was applied with rotation of both knobs simultaneously in counter clockwise direction. The entire procedure was done without touching the wall of antrum or pylorus. During the entry of insertion tube into the pyloric canal, a blurred image was seen due to contraction of pyloric wall around the tip of insertion tube. This finding is in accordance with the findings of Tams (1999). Lack of pyloric resistance and remarkable change in the color of mucosa from cream (of 
antrum and pylorus) to pinkish red (of duodenum) confirmed the entry of insertion tube into duodenum (Fig. 2). Further gentle advancement of tube with insufflations showed tunnel shaped descending duodenum. As the duodenum is uniform tubular structure, the further movement of insertion tube in duodenum was found easy. After crossing through proximal duodenal segment (Fig. 2), the major duodenal papilla (Fig. 3) was found at approximately $3-6 \mathrm{~cm}$ away from pylorus and it appeared as small thickened projection from the duodenal mucosa. The major duodenal papilla mostly located at seven o'clock on visualized field. The observation is in accordance with the reported findings of Jergens and Miles, (1994). A second papilla, the minor papilla became visible only in very few cases (Fig. 4). In duodenum the tube was easily passed by slight withdrawal of near portion of the endoscope. Slight withdrawal usually results into the straightening of insertion tube present in the stomach and forward movement of tip into duodenum (Fig. 5 and 6).
The endoscopic appearance of normal healthy duodenum from proximal duodenal segment to ascending duodenum in different sizes of dogs is given in Figure 1-6. The description for the same is given in Table 1 .

The color of mucosa of duodenum varied from pinkish red (38 cases) to yellowish red (22 cases) and it had velvety texture. Richter (1992) also recorded smooth and uniform duodenal mucosa with velvety texture on duodenum. Bile was noted in the duodenum in 16 cases, while sub mucosal blood vessels were visualized in two cases. However, erythema or erosion was not seen in any of the cases in this study. Richter (1992) and Jergens and Miles, (1994) also did not observed presence of erythema or erosions on duodenal mucosa of healthy dogs.

However Dowers et al., (2006) observed ulcers and Reimer et al., (1999) reported erosions and sub mucosal hemorrhages on the mucosa of duodenum in dogs and was considered as abnormal.

Table.1 Description of endoscopic appearance of normal healthy duodenum visualized in different cases

\begin{tabular}{|c|c|c|c|c|c|c|c|c|c|c|}
\hline \multirow[t]{2}{*}{$\begin{array}{l}\text { Endoscopic } \\
\text { Description }\end{array}$} & \multicolumn{2}{|c|}{ Color of mucous membrane } & \multicolumn{2}{|c|}{ Contents } & \multicolumn{2}{|c|}{ Erythema } & \multicolumn{2}{|c|}{ Erosion } & \multicolumn{2}{|c|}{$\begin{array}{l}\text { Sub mucosal } \\
\text { blood vessels }\end{array}$} \\
\hline & Pinkish red & Yellowish red & $\mathrm{P}$ & A & $\mathrm{P}$ & A & $\mathrm{P}$ & $\mathrm{A}$ & $\mathrm{P}$ & $\mathbf{A}$ \\
\hline $\begin{array}{l}\text { Number } \\
\text { of cases }\end{array}$ & 38 & 22 & $\begin{array}{c}16 \\
\text { bile }\end{array}$ & 44 & -- & 60 & $=$ & 60 & 2 & 58 \\
\hline
\end{tabular}

P: Present, A: Absent.

Table.2 Mean \pm SE distance $(\mathrm{cm})$ of different part/structures of upper digestive tract from upper incisors in different size of dog

\begin{tabular}{|l|l|l|l|l|}
$\begin{array}{l}\text { Sr. } \\
\text { No. }\end{array}$ & Part/structure & $\begin{array}{l}\text { Small } \\
\text { size }\end{array}$ & $\begin{array}{l}\text { Medium } \\
\text { size }\end{array}$ & $\begin{array}{l}\text { Large } \\
\text { size }\end{array}$ \\
\hline 1 & Pylorus & $54.17^{\mathrm{c}} \pm 1.25$ & $80.22^{\mathrm{b}} \pm 0.58$ & $\mathbf{9 3 . 5 7 ^ { \mathrm { a } } \pm 1 . 1 8}$ \\
\hline $\mathbf{2}$ & Major duodenal papilla & $60.52^{\mathrm{c}} \pm 1.28$ & $87.92^{\mathrm{b}} \pm 0.67$ & $98.75^{\mathrm{a}} \pm 0.86$ \\
\hline 3 & $\begin{array}{l}\text { Junction of descending and } \\
\text { ascending duodenum }\end{array}$ & $\mathbf{6 9 . 1 5 ^ { \mathrm { b } } \pm 1 . 3 9}$ & $100.85^{\mathrm{a}} \pm 0.56$ & $103.85^{\mathrm{a}} \pm 0.51$ \\
\hline
\end{tabular}

Means bearing same superscript in each row do not differ significantly $(\mathrm{P} \leq 0.05)$. 
Table.3 Distance $(\mathrm{cm})$ of pylorus and junction of descending and ascending duodenum from upper incisors and approximate length of insertion tube

\begin{tabular}{|l|l|l|c|c|}
\hline $\begin{array}{l}\text { Sr. } \\
\text { No. }\end{array}$ & Size of dog & Pylorus & $\begin{array}{l}\text { Junction of descending } \\
\text { and ascending } \\
\text { duodenum }\end{array}$ & $\begin{array}{c}\text { Approx. Length } \\
\text { of insertion tube } \\
\text { required (cm) }\end{array}$ \\
\hline $\mathbf{1}$ & Small size & $54.17 \pm 1.25$ & $69.15 \pm 1.39$ & 100 \\
\hline 2 & Medium size & $80.22 \pm 0.58$ & $100.85 \pm 0.56$ & 110 \\
\hline 3 & Large size & $\mathbf{9 3 . 5 7} \pm \mathbf{1 . 1 8}$ & $\mathbf{1 0 3 . 8 5} \pm \mathbf{0 . 5 0}$ & $\mathbf{1 1 0}-\mathbf{1 2 0}$ \\
\hline
\end{tabular}

\section{Endoscopic appearance of Duodenum}

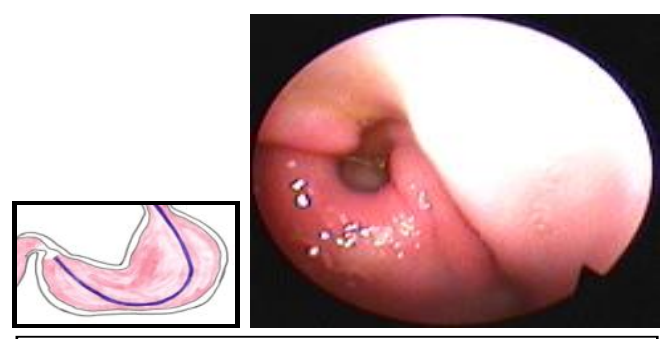

Fig. 1: Endoscopic appearance of open pylorus with approximate location of tube
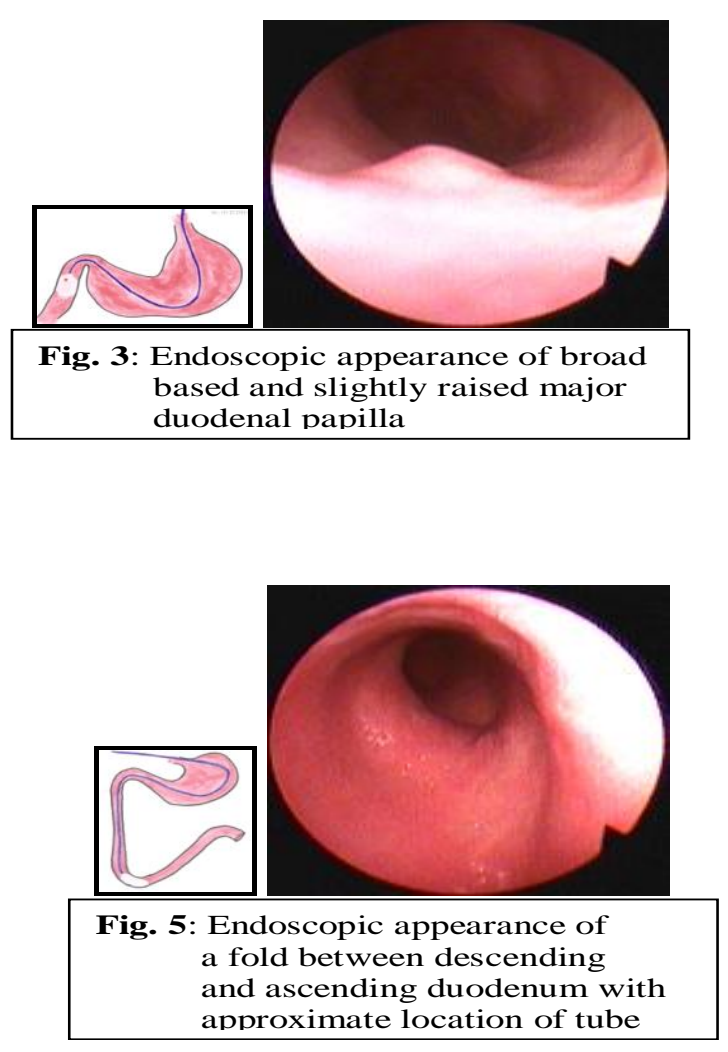

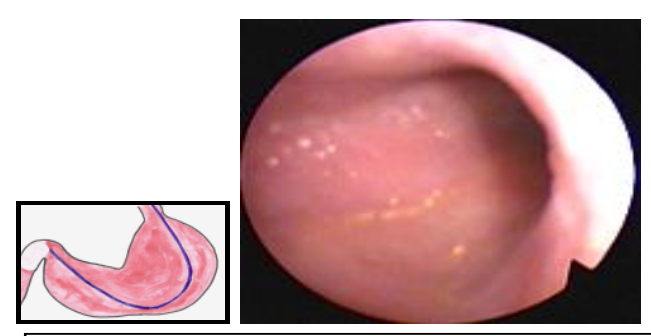

Fig. 2: Endoscopic appearance of proximal duodenal segment with approximate location of tube

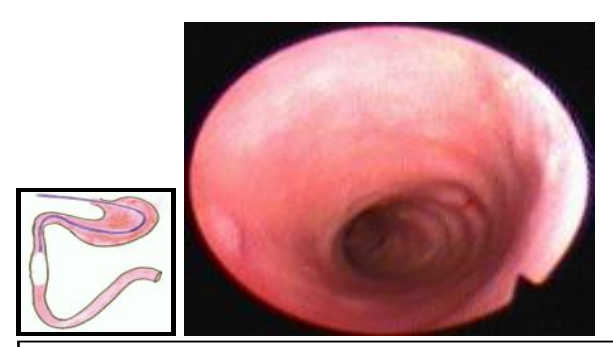

Fig. 4: Endoscopic appearance of descending duodenum \& minor papilla (at $8^{\circ}$ clock) with approximate location of tube

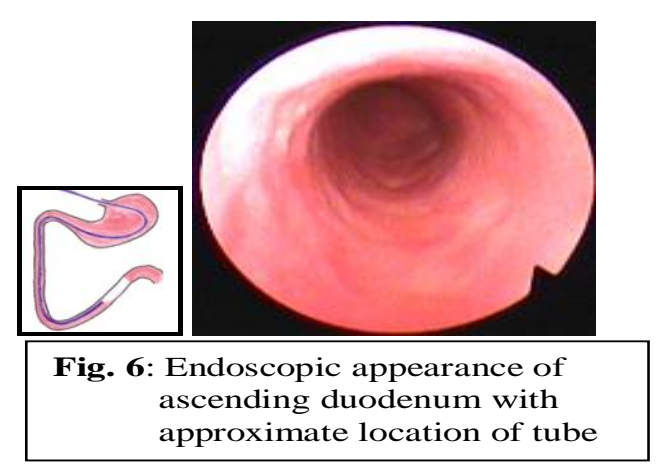


The knowledge of endoscopic findings of normal duodenum as well as applied knowledge of handling of endoscopic equipment was found essential for diagnosis of common abnormal cases of duodenum. Richter (1992) also opined that one must have the knowledge of proper endoscopic appearance of different structures for confirmation of diagnosis.

The duodenum was clearly visualized up to jejunum in maximum cases. The visualization of mucosa and its documentation was found better during the withdrawal stage of tube. Franz et al., (2004) also performed duodenoscopy for digital documentation of endoscopic view of normal duodenum in swine. Routine and daily practice of duodenoscopy of clinical cases was found essential in order to expertise in this technique. This technique was found as a less time consuming and hence could be advised to establish for the diagnosis of pyloric stenosis, pyloric and duodenal ulcers in clinical practice so as to provide correct therapy and faster recovery of the patient.

The distance of part from upper incisors was also studied in order to confirm the location of tip of insertion tube in different organs. It also helps in description of position and length of lesions as well as for detection of anatomical abnormalities by endoscopy (Strombeck and Guilford, 1991). The mean \pm SE distance $(\mathrm{cm})$ of different part/structures of upper digestive tract from upper incisors, in different sizes of dogs measured by endoscope is given in Table 2 .

The mean distance of Junction of descending and ascending duodenum from upper incisor was $69.15 \pm 1.39,100.85 \pm 0.56$ and $103.85 \pm$ $0.51 \mathrm{~cm}$ in small, medium and large sized dogs, respectively. The junction of descending and ascending duodenum significantly differed $(\mathrm{P}<0.05)$ in small sized dogs as compared to that in medium and large sized dogs but no significant difference was observed in medium and large sized dogs.

\section{Length of insertion tube required for different sizes of dogs}

The length of insertion tube required for gastroscopy varied in different sizes of dogs and it depended on the approximate distance of junction of descending and ascending duodenum from upper incisors. Depending on the distance of junction of descending and ascending duodenum from upper incisor, approximate length $(\mathrm{cm})$ of insertion tube required was calculated and is given in Table 3.

On the basis of above observations it could be said that working length of 100 and $110 \mathrm{~cm}$ would be suitable for small and medium sized dogs, respectively. The use of esophagogastroduodenoscope with working length of 110 to $120 \mathrm{~cm}$ would be more appropriate for large sized dogs to visualized digestive tract up to descending and ascending duodenum.

\section{References}

Dowers, K. L., S. R. Uhrig, K. R. Mama, J. S. Gaynor and P. W. Hellyer (2006). Effect of short-term sequential administration of non-steroidal antiinflammatory drugs on the stomach and proximal portion of the duodenum in healthy dogs. Am. J. Vet. Res. 67 (10):1794-801. [Abst: http://www. ncbiin/mm.nih. gov/pubmed/17014336].

Franz, S., H. Kahlbacher, M. Schuh and I. Schmerold (2004). Oesophagogastro duodenoscopy in swine--technique, methods, indications. Berl Munch Tierarztl Wochenschr. 117 (3-4): 153159. [Abst: http://www.ncbiin/m/nih. gov/pubmed]. 
Grooters, A. M., R. G. Sherding and S. E. Johnson (1994). Endoscopy case of the month: chronic vomiting and weight loss in a dog. Vet. Med. 89 (3): 196199.

Jergens, A. E. and K. G. Miles (1994). Endoscopy case of the month: chronic postprandial vomiting in a dog [chronic gastritis with a secondary gastric motility disorder]. Vet. Med. 89 (8): 754-755, 758-760.

Nicpon, J., K. Kubiak and G. Sapikowski (1999). Endoscopic evaluation of the influence of non-steroid antiinflammatory drugs on canine gastric mucosa. Medycyna Weterynaryjna. 55 (12): 809-811.
Reimer, M. E.; S. A. Johnston, M. S. Leib, Jr. R. B. Duncan, D. C. Reimer, M. Marini and K. Gimbert (1999). The gastroduodenal effects of buffered Aspirin, Carprofen, and Etodolac in healthy dogs. J. Vet. Int. Med. 13 (5): 472-477.

Richter, K. P. (1992). An introduction to endoscopy instrumentation and technique. Vet. Med. 87 (12): 1165, 1168-1171, 1174-1175.

Strombeck, D. R. and W. G. Guilford (1991). In: Small Animal Gastroenterology, $2^{\text {nd }}$ Ed. Wolfe Publishing Ltd. London: 119 - 128.

Tams, (1999) Small animal endoscopy, $2^{\text {nd }}$ Ed. Mosby, Inc. St. Louis, Missouri: 39 -179 and 247- 290.

\section{How to cite this article:}

Lokhande, D.U., L.B. Sarkate and Yadav, G.U. 2018. Endoscopic Evaluation of Normal Duodenum in 60 Healthy Dogs. Int.J.Curr.Microbiol.App.Sci. 7(11): 1334-1339.

doi: https://doi.org/10.20546/ijcmas.2018.711.155 